\title{
Probing the Quench Dynamics of Antiferromagnetic Correlations in a 2D Quantum Ising Spin System
}

\author{
Elmer Guardado-Sanchez, Peter T. Brown, Debayan Mitra, Trithep Devakul, \\ David A. Huse, Peter Schauß, and Waseem S. Bakr" \\ Department of Physics, Princeton University, Princeton, New Jersey 08544, USA
}

(Received 2 November 2017; revised manuscript received 1 May 2018; published 18 June 2018)

\begin{abstract}
Simulating the real-time evolution of quantum spin systems far out of equilibrium poses a major theoretical challenge, especially in more than one dimension. We experimentally explore quench dynamics in a two-dimensional Ising spin system with transverse and longitudinal fields. We realize the system with a near unit-occupancy atomic array of over 200 atoms obtained by loading a spin-polarized band insulator of fermionic lithium into an optical lattice and induce short-range interactions by direct excitation to a lowlying Rydberg state. Using site-resolved microscopy, we probe antiferromagnetic correlations in the system after a sudden quench from a paramagnetic state and compare our measurements to numerical calculations using state-of-the-art techniques. We achieve many-body states with longer-range antiferromagnetic correlations by implementing a near-adiabatic quench of the longitudinal field and study the buildup of correlations as we vary the rate with which we change the field.
\end{abstract}

DOI: 10.1103/PhysRevX.8.021069

Lattice quantum spin models serve as a paradigm for exploring a range of many-body phenomena, including quantum phase transitions [1,2], equilibration and thermalization $[3,4]$, and quench dynamics [5-10]. While there exists a variety of well-developed theoretical techniques to study the equilibrium properties of quantum spin systems [11-17], the toolkit for simulating real-time dynamics of these systems is rather limited and can only capture the evolution accurately for short times, especially for systems in more than one dimension [11,18-20]. Recent advances in the field of quantum simulation have introduced several experimental platforms where the dynamics of quantum spin systems can be measured over long evolution times, providing much needed benchmarks for testing uncontrolled theoretical approximations. Examples of such platforms include trapped ions [21-23], polar molecules [24], Rydberg atoms [25-29], magnetic atoms [30,31], and atoms interacting through superexchange in optical lattices [32-37].

In this work, we explore the dynamics of a two-dimensional quantum Ising model using a nearly defect-free array of

*wbakr@princeton.edu

Published by the American Physical Society under the terms of the Creative Commons Attribution 4.0 International license. Further distribution of this work must maintain attribution to the author(s) and the published article's title, journal citation, and DOI.

\author{
Subject Areas: Atomic and Molecular Physics, \\ Condensed Matter Physics, \\ Quantum Physics
}

neutral atoms which are coupled with laser light to a lowlying Rydberg state in an optical lattice [38]. The spin coupling in the model arises due to a van der Waals interaction between atoms in the Rydberg state. If one atom is in a Rydberg state, the excitation of another atom to a Rydberg state is strongly suppressed within a blockade radius $R_{b}$ [39-43]. This is because the interaction between the Rydberg atoms within this radius is much larger than the laser coupling strength. Previous experiments in $2 \mathrm{D}$ arrays have studied the regime $R_{b} \gg a_{l}$, where $a_{l}$ is the lattice spacing $[25,28]$. In this regime, the Rydberg blockade makes it difficult to access many-body states with a large Rydberg fraction. This significantly reduces the size of the relevant Hilbert space of the system from the maximum possible size of $2^{N}$, where $N$ is the number of sites, rendering simulation of the quantum dynamics feasible for the experimentally realized system sizes. Here, we focus on the regime $R_{b} \sim a_{l}$, where there is no such reduction of the Hilbert space size. This regime is particularly interesting because it features a direct quantum phase transition between a paramagnet and an antiferromagnet with broken $Z_{2}$ symmetry. Recently, Rydberg atoms in rearrangeable optical tweezers have explored this regime in 1D chains [29] and rings [28].

We realize a quantum Ising spin system with an array of ${ }^{6} \mathrm{Li}$ atoms in an optical lattice with near unit-occupancy. The lattice is deep enough to suppress tunneling over the timescale of the experiments. We prepare all the atoms in the same hyperfine ground state $|\downarrow\rangle$. Interactions are 
introduced by globally coupling the atoms with a single laser field to a Rydberg state $|\uparrow\rangle$. The van der Waals interaction between atoms in the Rydberg state is isotropic and takes the form $V_{i j}=C_{6} /\left|\mathbf{r}_{i}-\mathbf{r}_{j}\right|^{6}$. The Hamiltonian of the system is given by

$$
H=\hbar \Omega \sum_{i} \hat{S}_{i}^{x}+\sum_{i}\left(\mathcal{I}_{i}-\Delta\right) \hat{S}_{i}^{z}+\sum_{i \neq j} \frac{V_{i j}}{2} \hat{S}_{i}^{z} \hat{S}_{j}^{z}
$$

Here, $\hat{S}_{i}^{\alpha}$ are the spin $1 / 2$ operators for the $i$ th lattice site and $\alpha=x, y, z$. The first two terms of this Hamiltonian describe transverse and longitudinal magnetic fields that couple to the pseudospin. The Rabi frequency $\Omega$ that drives a transition between the ground and the Rydberg state for an isolated atom determines the transverse field, while the detuning $\Delta$ of the laser frequency from atomic resonance determines the longitudinal field. $\mathcal{I}_{i}=\sum_{j,(i \neq j)}\left(V_{i j} / 2\right)$ can be taken as a site-independent detuning in a large system as ours. We work with an attractively interacting $\left(V_{i j}<0\right)$ Rydberg state [44]. In the absence of the fields $(\Omega=0$ and $\mathcal{I}_{i}-\Delta=0$ ), the Hamiltonian's most excited state is a classical antiferromagnet. This state can be adiabatically connected to our initial state by slowly changing the fields. To achieve that, the starting state needs to be the most excited eigenstate of the initial Hamiltonian. For the rest of this paper, we find it convenient to invert the energy spectrum by introducing the Hamiltonian $\tilde{H}=-H$, so that we describe our work in a more familiar setting where the dynamics occurs close to the ground state of $\tilde{H}$ rather than the most excited state. For $R_{b}=\left(\left|C_{6}\right| / \hbar \Omega\right)^{1 / 6} \gg a_{l}$, the ground state phase diagram of $\tilde{H}$ in $\hbar \Omega-\Delta$ parameter space contains multiple Rydberg crystalline phases with different Rydberg atom fractions [29,45-49]. However, for $R_{b} \sim a_{l}$, the regime we study in this experiment, $\tilde{H}$, can be approximated by a nearest-neighbor Ising Hamiltonian with coupling $J=\left|C_{6}\right| / a_{l}^{6}$. A phase diagram for this model is shown in Fig. 1(a) and has only one ordered phase, the antiferromagnet [50-57]. The initial state in the experiment is the paramagnetic ground state of $\tilde{H}$ for positive detuning $\Delta \gg J \gg \hbar \Omega$. In this work, we quench the fields from this initial configuration to fields that support an antiferromagnetic ground state. We do this with varying degrees of adiabaticity and study the ensuing dynamics of the spin correlations.

We prepare nearly defect-free 2D arrays of atoms by taking advantage of Pauli blocking in a highly spinimbalanced degenerate Fermi gas loaded into a square optical lattice (for details, see Ref. [37]). The spin mixture consists of the first $|1\rangle$ and third $|3\rangle$ lowest hyperfine ground states of ${ }^{6} \mathrm{Li}$, with $|1\rangle$ as the majority. The minority atoms, needed to thermalize the gas while loading into the lattice, are subsequently removed with a pulse of resonant light. We focus our analysis on an annular region with outer (inner) radius of 9 (4) sites, where the average occupancy of

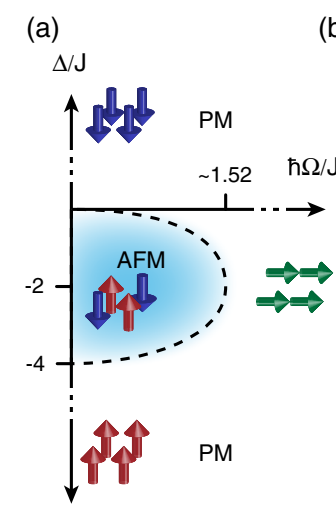

(b)

(c)

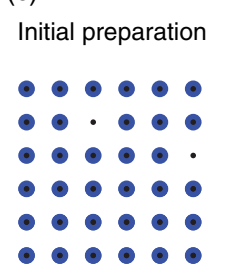

(d)

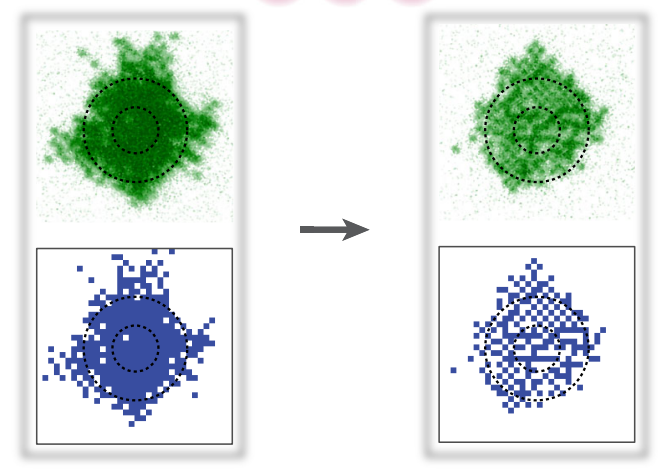

FIG. 1. Realization of a 2D quantum Ising model with Rydberg atoms in an optical lattice. (a) Ground-state phase diagram of the 2D quantum Ising model $\tilde{H}$, with nearest-neighbor coupling $J$. This is an approximate phase diagram of our Rydberg system when the blockade radius is comparable to the lattice spacing. Transverse and longitudinal fields are controlled by the Rabi frequency $\Omega$ and laser detuning $\Delta$, respectively. There is only one ordered phase, the antiferromagnet (AFM). Outside of this region there is a paramagnetic (PM) phase where the spins align with the field. (b) Experimental setup consisting of a 2D array of atoms at the focus of a high-resolution objective, capable of resolving individual sites of the lattice. Atoms in the ground state (small blue spheres) are directly coupled to the $23 P$ Rydberg state (large red spheres) with 230-nm laser light. (c) Typical atom configurations at different stages of the experiment. The initial state consists of an array of atoms in the electronic ground state (blue, left). This state is quenched into a state with antiferromagnetic correlations (Rydberg atoms in red, center). By increasing the lattice depth, Rydberg atoms are lost and only the ground state atoms are imaged (right). (d) Raw fluorescence images of an initial (left) and a postquench (right) configuration with strong antiferromagnetic correlations, together with reconstructed images (each blue pixel depicts a detected atom in the ground state). The annular region we used for analysis is shown delimited by dotted lines. 


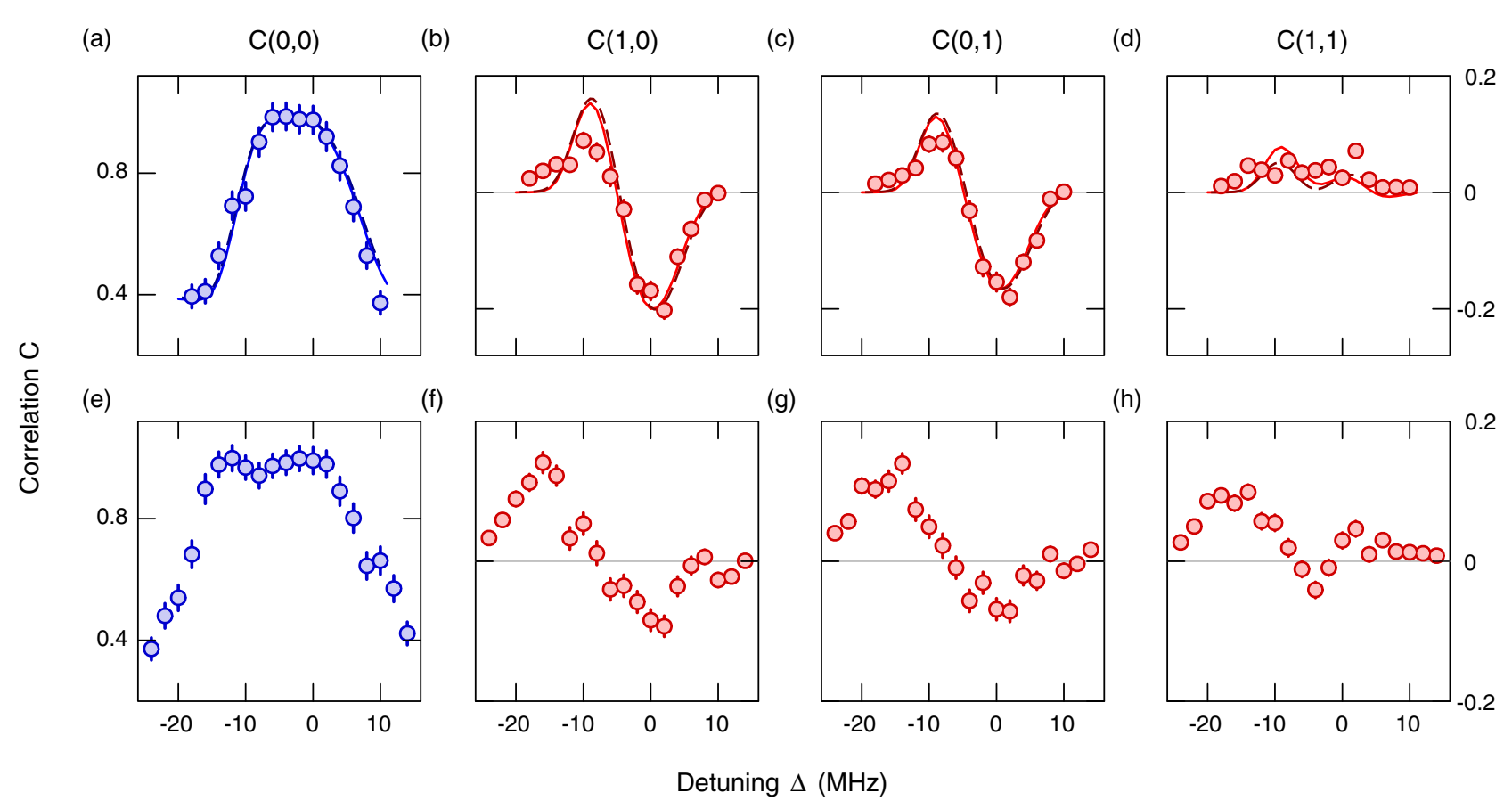

FIG. 2. Sudden quench dynamics. (a)-(d) Spin correlations after a sudden quench with $\Omega T=0.50(2) \pi[\Omega=2 \pi \times 4.1(1) \mathrm{MHz}]$ at various detunings $\Delta$. The correlators shown are (a) $C(0,0)$; (b) $C(1,0)$; (c) $C(0,1)$; and (d) $C(1,1)$. For comparison, we show the fits to dynamics computed with NLCE (solid line) and exact diagonalization on a $4 \times 4$ lattice with open boundary conditions (dashed line). (e)-(h) Spin correlations after a longer quench of $\Omega T=2.97(7) \pi[\Omega=2 \pi \times 5.3(1) \mathrm{MHz}]$ at various detunings.

the remaining state $|1\rangle$ atoms, measured from repeated preparations of the system, is maximal and corresponds to $95.7(4) \%$.

We couple the state $|1\rangle$ atoms to the $23 P\left(m_{l}=0\right.$, $\left.m_{s}=-1 / 2, m_{I}=1\right)$ Rydberg state using single-photon excitation with an ultraviolet (UV) laser at $230 \mathrm{~nm}$. The experiments are performed at a bias magnetic field of $595 \mathrm{G}$ pointing orthogonal to the 2D layer, allowing us to address a single $\left|m_{l}, m_{s}, m_{I}\right\rangle$ Rydberg state. Up to $60 \mathrm{~mW}$ of UV light is available from a frequency-quadrupled diode-laser system. The light is $\pi$-polarized and focused to a waist of $35 \mu \mathrm{m}$. The intensity and the frequency of the light can be changed rapidly to control the time dependence of the transverse and longitudinal fields in the Hamiltonian [44].

The atoms are located at the focus of a high resolution objective that can resolve individual sites of the optical lattice [Fig. 1(b)]. The Rydberg dynamics takes place in a lattice of depth $55 E_{R}$, where $E_{R}=(\pi \hbar)^{2} / 2 m a_{l}^{2}$ is the recoil energy and $a_{l}=1064 \mathrm{~nm} / \sqrt{2}$. We image the distribution of ground state atoms after removing Rydberg atoms with an efficiency of $90(3) \%$ by increasing the lattice depth to $2500 E_{R}$, leading to rapid photoionization or expulsion of the antitrapped Rydberg atoms [Fig. 1(c)]. We obtain siteresolved fluorescence images of the ground state atoms by collecting approximately 1000 photons per atom scattered from laser beams in a Raman cooling configuration [37].

We calibrate the transverse and longitudinal fields of the Hamiltonian using sparse clouds, where the average spacing between atoms is much larger than $R_{b}$. The location of the
Rydberg resonance $(\Delta=0)$ is determined by finding the laser frequency that maximizes atom loss during a long exposure to the UV light, since atoms in the Rydberg state experience an antitrapping optical potential. The Rabi frequency $\Omega$ is determined by measuring single atom Rabi oscillations, and we attain a maximum Rabi frequency $\Omega_{\max }=2 \pi \times 5.4(1) \mathrm{MHz}$ (the error bar takes into account shot-to-shot intensity fluctuations) [44]. $\Omega$ varies $4.9(3) \%$ over the region of interest due to the Gaussian intensity profile of the UV beam. The $C_{6}$ coefficient, which determines the strength of the van der Waals interaction, depends strongly on the principal quantum number. We obtain a theoretical $C_{6} / h=-1.92(6) \mathrm{MHz} \mu \mathrm{m}^{6}=-10.6(3) \mathrm{MHz}_{l}^{6}$ for the $23 P, m_{l}=0$ state at an offset field of $595 \mathrm{G}$ [44]. The angular dependence of the interaction potential in the $P$ state is unimportant in our experiments since the magnetic quantization axis is orthogonal to the plane of the lattice, leading to an isotropic interaction for atoms in the $2 \mathrm{D}$ plane. For these parameters, $\hbar \Omega_{\max }, J \gg h / \tau$, where $\tau \sim 20 \mu$ s is the lifetime of the Rydberg state [58], leading to negligible decay over the relevant timescales.

We first study dynamics in the Ising system after a sudden quench, where the transverse field is switched on quickly compared to $\Omega^{-1}$. The system is initially in a product state, with all spins in $|\downarrow\rangle$, and we image the atoms after an evolution time $T$. From the images, we extract the spin correlators $C(\mathbf{r})=4\left\langle\hat{S}_{\mathbf{i}}^{z} \hat{S}_{\mathbf{i}+\mathbf{r}}^{z}\right\rangle_{c}=4\left(\left\langle\hat{S}_{\mathbf{i}}^{z} \hat{S}_{\mathbf{i}+\mathbf{r}}^{z}\right\rangle-\left\langle\hat{S}_{\mathbf{i}}^{z}\right\rangle\left\langle\hat{S}_{\mathbf{i}+\mathbf{r}}^{z}\right\rangle\right)$. The correlators $C(0,0) ; C(1,0) ; C(0,1) ;$ and $C(1,1)$ are shown in Figs. 2(a)-2(d) for $\Omega T=0.50(2) \pi$ 

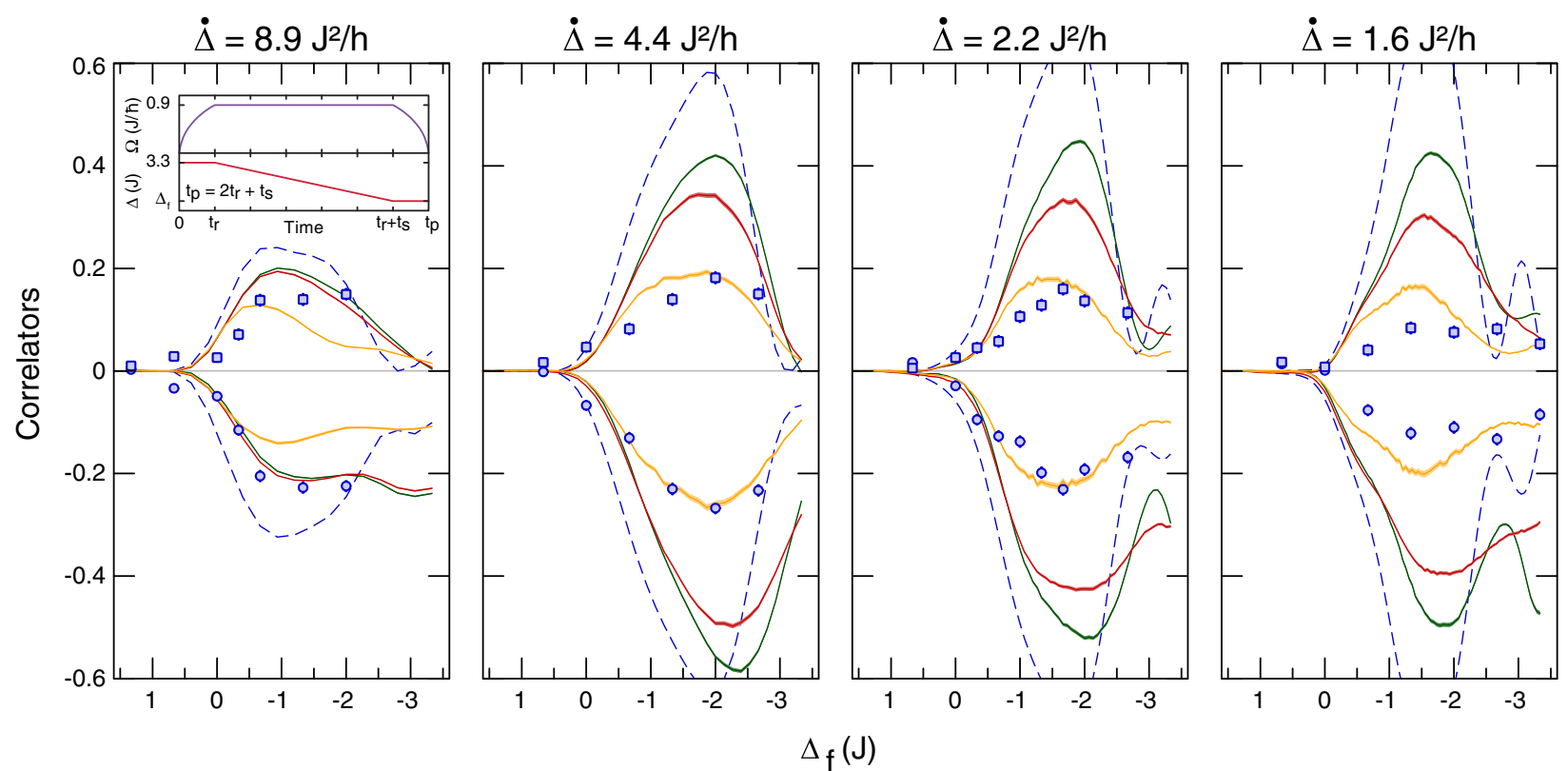

$\Delta_{f}(J)$

FIG. 3. Time evolution of spin correlations after near-adiabatic quenches and comparison with phenomenological decoherence models: experimental correlations after near-adiabatic quenches [blue circles for $C(0,1)$ and blue squares for $C(1,1)$ ] for different quench rates (left to right panels) and varying final detuning $\Delta_{f}$. These are compared with MCWF simulations taking into account different types of decoherence (lines). We studied $T_{1}$ type decoherence with $T_{1}=20 \mu \mathrm{s}$ (green) and $T_{2}$ type decoherence with $T_{2}=0.5 \mu \mathrm{s}$ (red). The $T_{1}$ value is chosen as the lifetime of the Rydberg state and the $T_{2}$ value is an aggressive lower bound given by our Ramsey calibration [44]. Additionally, we show the combination of $T_{1}$ decoherence with two-particle "interaction noise" for nearestneighbor pairs (yellow). For the latter, the decoherence rate $\left(\Gamma_{J}=1 \mu \mathrm{s}^{-1}\right)$ was a free parameter chosen to obtain reasonable agreement with experimental correlations for all four quenches simultaneously [44]. The shaded regions depict the standard error of the mean of the simulations. For reference, the calculations without decoherence are also shown (dashed blue lines). Inset: Time dependence of the Rabi frequency $\Omega$ and detuning $\Delta$ used for the near-adiabatic quenches. The time for switching on and off the laser coupling was fixed to $t_{r}=0.6 \mathrm{~h} / \mathrm{J}$ for all quenches. The maximum total length of the quench $t_{p}$ varies from 0.3 to $0.9 \mu \mathrm{s}$, depending on the quench rate.

$(\Omega=2 \pi \times 4.1(1) \mathrm{MHz})$ and varying detuning $\Delta$. The correlator $C(0,0)$ is linked to the magnetization as $C(0,0)=1-4\left\langle\hat{S}_{\mathbf{i}}^{z}\right\rangle^{2}$. We observe a change in the sign of the nearest-neighbor correlations as the detuning $\Delta$ is varied.

For such short times, the correlations remain short range and, therefore, we can compare our results to calculations obtained using a dynamical version $[44,59]$ of the numerical linked cluster expansion (NLCE) [16,60]. The dynamics is computed on clusters of increasing size (the "order" of the expansion), and the results are expected to converge if the correlation length is smaller than the cluster size. We find good convergence for times $\Omega T \lesssim \pi$ [44]. The 11th order NLCE results for the on-site and nearest-neighbor correlations are fit to the measured correlations after the quench with two free parameters: the van der Waals interaction coefficient $C_{6}$ and a scaling factor $\alpha$ corresponding to the Rydberg imaging efficiency. The NLCE dynamics calculations take into account interactions up to next-nearest neighbors and experimental imperfections including the finite rise and fall time of $\Omega$ and $2.8 \%$ anisotropy of the lattice spacing [37], which translates to an $18 \%$ anisotropy of the interactions on the nearest-neighbor sites. We also compare the data to exact diagonalization results on a $4 \times 4$ lattice. From these fits, we obtain an experimental $\quad C_{6} / h=-1.1(1) \mathrm{MHz} \mu \mathrm{m}^{6}=-6.0(3) \mathrm{MHz}_{l}^{6}$ and a scaling factor $\alpha=0.89(1)$, which agrees with the expected detection efficiency [44]. The fitted value of $C_{6}$ is about $40 \%$ lower than the theoretically calculated $C_{6}$, which has possible systematic errors due to uncertainties in the matrix elements in lithium, in particular at high magnetic fields, and finite wave function size of the atoms on the lattice sites $[44,61]$.

To go beyond the regime where the dynamics can be calculated with NLCE, we perform a longer quench with $\Omega T=2.97(7) \pi$. The extracted correlators are shown in Figs. 2(e)-2(h). In this case, even the next-nearest-neighbor correlations exhibit a zero crossing as a function of detuning, showing that the system is building up longerrange correlations. The different NLCE orders already stop converging at much earlier times [44].

To prepare many-body states with longer antiferromagnetic correlations, we investigate a more adiabatic quench scheme $[48,62,63]$, illustrated in the inset of Fig. 3. In the following, we use $J=h \times 6 \mathrm{MHz}$ for all presented units. We 
start from the same initial state but use a soft switch on and off of the Rabi frequency and a linear ramp of the detuning from $\Delta_{i}=3.3 \mathrm{~J}$ to a varying $\Delta_{f}$. During the detuning ramp, the Rabi frequency is fixed at $\Omega_{0}=0.9(1) J / \hbar$. We explore a variety of detuning ramp rates $\dot{\Delta}$, ranging from $8.9 \mathrm{~J}^{2} / h$ to $1.6 J^{2} / h$. For each $\dot{\Delta}$, we measure correlations at different times in the ramp. Figure 3 shows the buildup of nearestneighbor and next-nearest-neighbor antiferromagnetic correlations as the longitudinal field is ramped at different rates. The buildup of antiferromagnetic correlations starts approximately at the time the detuning ramp crosses $\Delta=0$. For the fastest quench rates, we see a correlation buildup before crossing of the resonance, which we attribute to strong nonadiabaticities. For all quench rates studied, we observe that the correlations reach a maximum at $\Delta / J \sim-2$, as would be expected in the adiabatic limit from the phase diagram in Fig. 1(a). The peak value of the correlations initially increases as the quench rate is reduced, as one might expect for approaching the adiabatic limit, but then decreases for slower ramps. This is likely due to decoherence starting to play a role in the slower quenches. Therefore, we have to take decoherence effects into account for a numerical modeling of the time dependence of the correlations.

We performed a phenomenological study of the influence of decoherence on the near-adiabatic ramps by solving the master equation using the Monte Carlo wave function method (MCWF) on a $4 \times 4$ lattice $[64,65]$. Single-particle decoherence comes in the form of decay from the Rydberg state $\left(T_{1}\right)$ and dephasing that can be characterized in our system using a Ramsey sequence in a sparse cloud $\left(T_{2}\right)$ [44]. In Fig. 3, we show MCWF simulations with values of $T_{1}=20 \mu \mathrm{s}$ and $T_{2}=0.5 \mu \mathrm{s}$. We found that the impact of single-particle decoherence on the correlations is too small to reproduce our experimental results. However, motional effects can lead to many-body decoherence. Mechanisms leading to atomic motion include strong attractive forces between atoms in the Rydberg state, laser recoil, and changes of the lattice potential experienced by the atoms due to a difference in the polarizability between the ground and Rydberg states. These motional effects are stronger in our system compared to previous optical tweezer experiments [28,29] due to the light mass of lithium and the relatively small lattice spacing. This motion of the atoms, estimated in Ref. [44], leads to decoherence in the spin system by changing the coupling $J$. To model this twoparticle decoherence, we approximated the movement of the atoms as "interaction noise" between nearest-neighbor pairs. Although the motion is expected to be coherent at short times, we make the assumption that the motion is sufficiently chaotic at long times to allow us to use this decoherence approximation. We implement the "interaction noise" by adding a time-independent conditional nearestneighbor dephasing term with rate $\Gamma_{J}=1 \mu \mathrm{s}^{-1}$ to the Lindblad master equation [44]. As the "interaction noise" is not constrained by our single-particle calibrations, we use its strength as a single free parameter to fit our data. This phenomenological simulation allows us to achieve better agreement with our data for the longer quench rates, suggesting that our main source of decoherence is two- or many-body in nature. A more detailed discussion of the modeling of decoherence effects can be found in Ref. [44].

At the end of the ramps, where significant antiferromagnetic correlations have built up, we find that we can fit the decay of the correlations with distance to an exponential [Fig. 4(a)]. The fitted correlation lengths range from $\xi=0.74(6) a_{l}$ to $\xi=1.9(2) a_{l}$ depending on $\dot{\Delta}$. We compared these data to the results of equilibrium Monte Carlo calculations at $\Delta_{f}=-2 J$ and $\Omega=0$ to check if we can describe the system in terms of an effective temperature. While these calculations also give correlations that decay exponentially, there is no temperature that reproduces

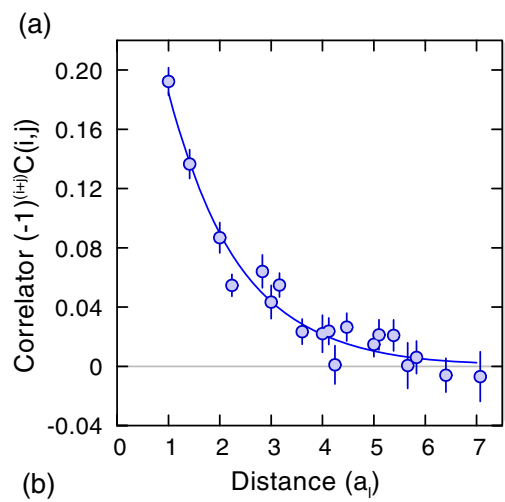

(c)

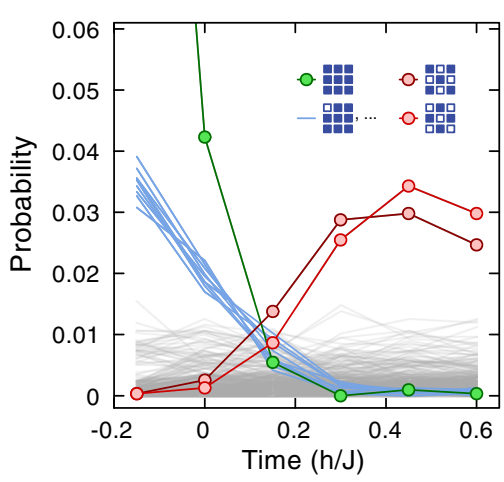

$\dot{\Delta}=2.2 \mathrm{~J}^{2} / \mathrm{h}$
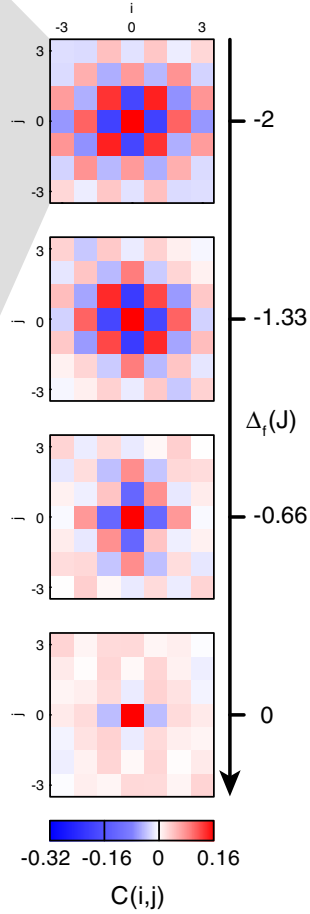

FIG. 4. Characterizing many-body states during and after a slow quench. (a) Spatial decay of the correlations after a sweep with $\dot{\Delta}=2.2 \mathrm{~J}^{2} / h$, with an exponential fit that yields a correlation length $\xi=1.4(1) a_{l}$. (b) Time evolution of the probabilities of observing different configurations in $3 \times 3$ subsystems, not corrected for detection efficiencies. The probabilities are shown for the two antiferromagnetic states (red), the all-grounds state (green), one-Rydberg-atom states (blue), and all other states (grey). The evolution is shown during a ramp with $\dot{\Delta}=4.4 J^{2} / h$. The antiferromagnetic configurations become most probable at the end of the quench. (c) Full correlation matrices $C(i, j)$ at different final detunings during a slow quench with $\dot{\Delta}=2.2 J^{2} / h$, showing the growing range of the antiferromagnetic correlations. 
both the strength and range of the correlations. We find a temperature of $k_{B} T_{\xi} \approx 0.51(1) \mathrm{J}$ for matching the correlation length and $k_{B} T_{n n} \approx 0.82(4) J$ when matching the nearest-neighbor correlator for the data shown in Fig. 4(a) (for details, see Ref. [44]). The fact that these two temperatures are not within error bars leads us to the conclusion that the system is not equilibrated at the end of the quench.

Another way to characterize the states created by these slow quenches is by extracting the probabilities for observing a particular spin configuration in a subsystem. In Fig. 4(b), we show the probability of observing different spin configurations in $3 \times 3$ subsystems, not correcting for detection fidelities. The two antiferromagnetic states are the most probable states near the end of the ramp, with an enhancement of a factor of 16(2) over a uniform distribution in the Hilbert space.

In conclusion, we studied quench dynamics in a 2D Ising model realized with ultracold atoms coupled to a Rydberg state in an optical lattice. The use of a light fermionic atom, ${ }^{6} \mathrm{Li}$, allows us to use Pauli blocking in a relatively large spacing lattice to create $2 \mathrm{D}$ atomic arrays with high filling (approximately 96\%), comparable to what is achieved in atom-by-atom assembler experiments [66,67]. Combining the large spacing with the use of a low-lying Rydberg state, we reached the strong correlation regime with $R_{b} \sim a_{l}$ and prepared states exhibiting strong short-range antiferromagnetic correlations. We found good agreement of our data with state-of-the-art numerics for short-time quench dynamics without taking into account decoherence. In our study of near-adiabatic quenches, we obtained evidence for beyond single-particle decoherence in our system and observed nonequilibrated final states with longer-range antiferromagnetic correlations. Our new ultracold ${ }^{6} \mathrm{Li}$ Rydberg platform opens many interesting directions for future work. Rydberg excitation in a Fermi gas may allow the exploration of impurity dynamics in the presence of Pauli blocking effects $[68,69]$. Another possible direction is the use of Rydberg dressing techniques to realize a dipolar Fermi gas, taking advantage of the fast tunneling of lithium in an optical lattice to go beyond the frozen gas regime [27,70-72].

This work was supported by the NSF (Grant No. DMR1607277), the David and Lucile Packard Foundation (Grant No. 2016-65128), and the AFOSR Young Investigator Research Program (Grant No. FA9550-16-1-0269). W. S. B. was supported by an Alfred P. Sloan Foundation fellowship. P. T. B. was supported by the DoD through the NDSEG Fellowship Program.

Note added.-Recently, antiferromagnetic correlations have been observed in 2D arrays of Rydberg atoms trapped in optical tweezers in experiments at the Institut d'Optique by Vincent Lienhard et al. [73].
[1] S. Sachdev, Quantum Phase Transitions (Cambridge University Press, Cambridge, England, 1999).

[2] S. Tanaka, R. Tamura, and B. K. Chakrabarti, Quantum Spin Glasses, Annealing and Computation (Cambridge University Press, Cambridge, England, 2017).

[3] B. Blaß and H. Rieger, Test of Quantum Thermalization in the Two-Dimensional Transverse-Field Ising Model, Sci. Rep. 6, 38185 (2016).

[4] R. Mondaini, K. R. Fratus, M. Srednicki, and M. Rigol, Eigenstate Thermalization in the Two-Dimensional Transverse Field Ising Model, Phys. Rev. E 93, 032104 (2016).

[5] J. Eisert, M. Friesdorf, and C. Gogolin, Quantum ManyBody Systems out of Equilibrium, Nat. Phys. 11, 124 (2015).

[6] K. Sengupta, S. Powell, and S. Sachdev, Quench Dynamics across Quantum Critical Points, Phys. Rev. A 69, 053616 (2004).

[7] P. Barmettler, M. Punk, V. Gritsev, E. Demler, and E. Altman, Relaxation of Antiferromagnetic Order in Spin-1/2 Chains Following a Quantum Quench, Phys. Rev. Lett. 102, 130603 (2009).

[8] H. Rieger and F. Iglói, Semiclassical Theory for Quantum Quenches in Finite Transverse Ising Chains, Phys. Rev. B 84, 165117 (2011).

[9] P. Calabrese, F. H. L. Essler, and M. Fagotti, Quantum Quench in the Transverse-Field Ising Chain, Phys. Rev. Lett. 106, 227203 (2011).

[10] N. O. Abeling and S. Kehrein, Quantum Quench Dynamics in the Transverse Field Ising Model at Nonzero Temperatures, Phys. Rev. B 93, 104302 (2016).

[11] Strongly Correlated Systems, Springer Series in Solid-State Sciences, edited by A. Avella and F. Mancini (SpringerVerlag, Berlin, Heidelberg, 2013).

[12] W. von der Linden, A Quantum Monte Carlo Approach to Many-Body Physics, Phys. Rep. 220, 53 (1992).

[13] S. R. White, Density Matrix Formulation for Quantum Renormalization Groups, Phys. Rev. Lett. 69, 2863 (1992).

[14] U. Schollwöck, The Density-Matrix Renormalization Group, Rev. Mod. Phys. 77, 259 (2005).

[15] K. A. Hallberg, New Trends in Density Matrix Renormalization, Adv. Phys. 55, 477 (2006).

[16] B. Tang, E. Khatami, and M. Rigol, A Short Introduction to Numerical Linked-Cluster Expansions, Comput. Phys. Commun. 184, 557 (2013).

[17] R. Orús, A Practical Introduction to Tensor Networks: Matrix Product States and Projected Entangled Pair States, Ann. Phys. (Amsterdam) 349, 117 (2014).

[18] G. Vidal, Efficient Simulation of One-Dimensional Quantum Many-Body Systems, Phys. Rev. Lett. 93, 040502 (2004).

[19] S. R. White and A. E. Feiguin, Real-Time Evolution Using the Density Matrix Renormalization Group, Phys. Rev. Lett. 93, 076401 (2004).

[20] K. R. A. Hazzard, M. van den Worm, M. Foss-Feig, S. R. Manmana, E. G. D. Torre, T. Pfau, M. Kastner, and A. M. Rey, Quantum Correlations and Entanglement in Far-fromEquilibrium Spin Systems, Phys. Rev. A 90, 063622 (2014).

[21] K. Kim, M.-S. Chang, S. Korenblit, R. Islam, E. E. Edwards, J. K. Freericks, G.-D. Lin, L.-M. Duan, and C. Monroe, Quantum Simulation of Frustrated Ising Spins with Trapped Ions, Nature (London) 465, 590 (2010). 
[22] J. W. Britton, B. C. Sawyer, A. C. Keith, C.-C. J. Wang, J. K. Freericks, H. Uys, M. J. Biercuk, and J. J. Bollinger, Engineered Two-Dimensional Ising Interactions in a Trapped-Ion Quantum Simulator with Hundreds of Spins, Nature (London) 484, 489 (2012).

[23] J. G. Bohnet, B. C. Sawyer, J. W. Britton, M. L. Wall, A. M. Rey, M. Foss-Feig, and J. J. Bollinger, Quantum Spin Dynamics and Entanglement Generation with Hundreds of Trapped Ions, Science 352, 1297 (2016).

[24] B. Yan, S. A. Moses, B. Gadway, J. P. Covey, K. R. A. Hazzard, A. M. Rey, D. S. Jin, and J. Ye, Observation of Dipolar Spin-Exchange Interactions with Lattice-Confined Polar Molecules, Nature (London) 501, 521 (2013).

[25] P. Schauß, M. Cheneau, M. Endres, T. Fukuhara, S. Hild, A. Omran, T. Pohl, C. Gross, S. Kuhr, and I. Bloch, Observation of Spatially Ordered Structures in a TwoDimensional Rydberg Gas, Nature (London) 491, 87 (2012).

[26] K. M. Maller, M. T. Lichtman, T. Xia, Y. Sun, M. J. Piotrowicz, A. W. Carr, L. Isenhower, and M. Saffman, Rydberg-Blockade Controlled-Not Gate and Entanglement in a Two-Dimensional Array of Neutral-Atom Qubits, Phys. Rev. A 92, 022336 (2015).

[27] Y.-Y. Jau, A. M. Hankin, T. Keating, I. H. Deutsch, and G. W. Biedermann, Entangling Atomic Spins with a RydbergDressed Spin-Flip Blockade, Nat. Phys. 12, 71 (2016).

[28] H. Labuhn, D. Barredo, S. Ravets, S. de Léséleuc, T. Macrí, T. Lahaye, and A. Browaeys, Tunable Two-Dimensional Arrays of Single Rydberg Atoms for Realizing Quantum Ising Models, Nature (London) 534, 667 (2016).

[29] H. Bernien, S. Schwartz, A. Keesling, H. Levine, A. Omran, H. Pichler, S. Choi, A. S. Zibrov, M. Endres, M. Greiner, V. Vuletic, and M. D. Lukin, Probing Many-Body Dynamics on a 51-Atom Quantum Simulator, Nature (London) 551, 579 (2017).

[30] A. de Paz, A. Sharma, A. Chotia, E. Maréchal, J.H. Huckans, P. Pedri, L. Santos, O. Gorceix, L. Vernac, and B. Laburthe-Tolra, Nonequilibrium Quantum Magnetism in a Dipolar Lattice Gas, Phys. Rev. Lett. 111, 185305 (2013).

[31] S. Baier, M. J. Mark, D. Petter, K. Aikawa, L. Chomaz, Z. Cai, M. Baranov, P. Zoller, and F. Ferlaino, Extended BoseHubbard Models with Ultracold Magnetic Atoms, Science 352, 201 (2016).

[32] T. Fukuhara, A. Kantian, M. Endres, M. Cheneau, P. Schauß, S. Hild, D. Bellem, U. Schollwöck, T. Giamarchi, C. Gross, I. Bloch, and S. Kuhr, Quantum Dynamics of a Mobile Spin Impurity, Nat. Phys. 9, 235 (2013).

[33] M. Boll, T. A. Hilker, G. Salomon, A. Omran, J. Nespolo, L. Pollet, I. Bloch, and C. Gross, Spin- and Density-Resolved Microscopy of Antiferromagnetic Correlations in FermiHubbard Chains, Science 353, 1257 (2016).

[34] L. W. Cheuk, M. A. Nichols, K. R. Lawrence, M. Okan, H. Zhang, E. Khatami, N. Trivedi, T. Paiva, M. Rigol, and M. W. Zwierlein, Observation of Spatial Charge and Spin Correlations in the 2D Fermi-Hubbard Model, Science 353, 1260 (2016).

[35] M. F. Parsons, A. Mazurenko, C. S. Chiu, G. Ji, D. Greif, and M. Greiner, Site-Resolved Measurement of the SpinCorrelation Function in the Fermi-Hubbard Model, Science 353, 1253 (2016).
[36] J. H. Drewes, L. A. Miller, E. Cocchi, C. F. Chan, N. Wurz, M. Gall, D. Pertot, F. Brennecke, and M. Köhl, Antiferromagnetic Correlations in Two-Dimensional Fermionic Mott-Insulating and Metallic Phases, Phys. Rev. Lett. 118, 170401 (2017).

[37] P. T. Brown, D. Mitra, E. Guardado-Sanchez, P. Schauß, S. S. Kondov, E. Khatami, T. Paiva, N. Trivedi, D. A. Huse, and W.S. Bakr, Spin-Imbalance in a 2D Fermi-Hubbard System, Science 357, 1385 (2017).

[38] T.F. Gallagher, Rydberg Atoms (Cambridge University Press, Cambridge, England, 1994).

[39] K. Singer, M. Reetz-Lamour, T. Amthor, L. G. Marcassa, and M. Weidemüller, Suppression of Excitation and Spectral Broadening Induced by Interactions in a Cold Gas of Rydberg Atoms, Phys. Rev. Lett. 93, 163001 (2004).

[40] D. Tong, S. M. Farooqi, J. Stanojevic, S. Krishnan, Y. P. Zhang, R. Côté, E. E. Eyler, and P. L. Gould, Local Blockade of Rydberg Excitation in an Ultracold Gas, Phys. Rev. Lett. 93, 063001 (2004).

[41] R. Heidemann, U. Raitzsch, V. Bendkowsky, B. Butscher, R. Löw, and T. Pfau, Rydberg Excitation of Bose-Einstein Condensates, Phys. Rev. Lett. 100, 033601 (2008).

[42] E. Urban, T. A. Johnson, T. Henage, L. Isenhower, D. D. Yavuz, T. G. Walker, and M. Saffman, Observation of Rydberg Blockade between Two Atoms, Nat. Phys. 5, 110 (2009).

[43] A. Gaëtan, Y. Miroshnychenko, T. Wilk, A. Chotia, M. Viteau, D. Comparat, P. Pillet, A. Browaeys, and P. Grangier, Observation of Collective Excitation of Two Individual Atoms in the Rydberg Blockade Regime, Nat. Phys. 5, 115 (2009).

[44] See the Supplemental Material http://link.aps.org/ supplemental/10.1103/PhysRevX.8.021069 for details of the experimental setup, characterization of single-particle decoherence and additional information on the used theoretical techniques.

[45] P. Fendley, K. Sengupta, and S. Sachdev, Competing Density-Wave Orders in a One-Dimensional Hard-Boson Model, Phys. Rev. B 69, 075106 (2004).

[46] H. Weimer, R. Löw, T. Pfau, and H. P. Büchler, Quantum Critical Behavior in Strongly Interacting Rydberg Gases, Phys. Rev. Lett. 101, 250601 (2008).

[47] H. Weimer and H. P. Büchler, Two-Stage Melting in Systems of Strongly Interacting Rydberg Atoms, Phys. Rev. Lett. 105, 230403 (2010).

[48] T. Pohl, E. Demler, and M. D. Lukin, Dynamical Crystallization in the Dipole Blockade of Ultracold Atoms, Phys. Rev. Lett. 104, 043002 (2010).

[49] E. Sela, M. Punk, and M. Garst, Dislocation-Mediated Melting of One-Dimensional Rydberg Crystals, Phys. Rev. B 84, 085434 (2011).

[50] A. Yanase, Y. Takeshige, and M. Suzuki, Critical Behaviour of the Susceptibility of the Ising Model with a Transverse Field, J. Phys. Soc. Jpn. 41, 1108 (1976).

[51] C. J. Hamer and A. C. Irving, Cluster Expansions in the $(2+1)$ D Ising Model, J. Phys. A 17, 1649 (1984).

[52] T. E. Lee, H. Häffner, and M. C. Cross, Antiferromagnetic Phase Transition in a Nonequilibrium Lattice of Rydberg Atoms, Phys. Rev. A 84, 031402 (2011). 
[53] S. Ji, C. Ates, and I. Lesanovsky, Two-Dimensional Rydberg Gases and the Quantum Hard-Squares Model, Phys. Rev. Lett. 107, 060406 (2011).

[54] I. Lesanovsky, Many-Body Spin Interactions and the Ground State of a Dense Rydberg Lattice Gas, Phys. Rev. Lett. 106, 025301 (2011).

[55] J. Qian, G. Dong, L. Zhou, and W. Zhang, Phase Diagram of Rydberg Atoms in a Nonequilibrium Optical Lattice, Phys. Rev. A 85, 065401 (2012).

[56] A. Hu, T. E. Lee, and C. W. Clark, Spatial Correlations of One-Dimensional Driven-Dissipative Systems of Rydberg Atoms, Phys. Rev. A 88, 053627 (2013).

[57] M. Hoening, W. Abdussalam, M. Fleischhauer, and T. Pohl, Antiferromagnetic Long-Range Order in Dissipative Rydberg Lattices, Phys. Rev. A 90, 021603 (2014).

[58] I. I. Beterov, I. I. Ryabtsev, D. B. Tretyakov, and V. M. Entin, Quasiclassical Calculations of BlackbodyRadiation-Induced Depopulation Rates and Effective Lifetimes of Rydberg $n s, n p$, and nd Alkali-Metal Atoms with $n \leq 80$, Phys. Rev. A 79, 052504 (2009).

[59] I. G. White, B. Sundar, and K. R. A. Hazzard, Quantum Dynamics from a Numerical Linked Cluster Expansion, arXiv:1710.07696.

[60] M. Rigol, T. Bryant, and R. R. P. Singh, Numerical LinkedCluster Algorithms. I. Spin Systems on Square, Triangular, and Kagomé Lattices, Phys. Rev. E 75, 061118 (2007).

[61] M. L. Wall and L. D. Carr, Dipole-Dipole Interactions in Optical Lattices Do Not Follow an Inverse Cube Power Law, New J. Phys. 15, 123005 (2013).

[62] J. Schachenmayer, I. Lesanovsky, A. Micheli, and A. J. Daley, Dynamical Crystal Creation with Polar Molecules or Rydberg Atoms in Optical Lattices, New J. Phys. 12, 103044 (2010).

[63] R. M. W. van Bijnen, S. Smit, K. a. H. van Leeuwen, E. J. D. Vredenbregt, and S. J. J. M. F. Kokkelmans, Adiabatic Formation of Rydberg Crystals with Chirped Laser Pulses, J. Phys. B 44, 184008 (2011).
[64] K. Mølmer, Y. Castin, and J. Dalibard, Monte Carlo WaveFunction Method in Quantum Optics, J. Opt. Soc. Am. B 10, 524 (1993).

[65] H. Weimer, Tailored Jump Operators for Purely Dissipative Quantum Magnetism, J. Phys. B 50, 024001 (2017).

[66] D. Barredo, S. de Léséleuc, V. Lienhard, T. Lahaye, and A. Browaeys, An Atom-by-Atom Assembler of Defect-Free Arbitrary Two-Dimensional Atomic Arrays, Science 354, 1021 (2016).

[67] M. Endres, H. Bernien, A. Keesling, H. Levine, E. R. Anschuetz, A. Krajenbrink, C. Senko, V. Vuletic, M. Greiner, and M.D. Lukin, Atom-by-Atom Assembly of Defect-Free One-Dimensional Cold Atom Arrays, Science 354, 1024 (2016).

[68] A. Gaj, A. T. Krupp, J. B. Balewski, R. Löw, S. Hofferberth, and T. Pfau, From Molecular Spectra to a Density Shift in Dense Rydberg Gases, Nat. Commun. 5, 5546 (2014).

[69] R. Schmidt, H. R. Sadeghpour, and E. Demler, Mesoscopic Rydberg Impurity in an Atomic Quantum Gas, Phys. Rev. Lett. 116, 105302 (2016).

[70] B. Xiong, H. H. Jen, and D.-W. Wang, Topological Superfluid by Blockade Effects in a Rydberg-Dressed Fermi Gas, Phys. Rev. A 90, 013631 (2014).

[71] X. Li and S. D. Sarma, Exotic Topological Density Waves in Cold Atomic Rydberg-Dressed Fermions, Nat. Commun. 6, 7137 (2015).

[72] J. Zeiher, R. van Bijnen, P. Schauß, S. Hild, J.-y. Choi, T. Pohl, I. Bloch, and C. Gross, Many-Body Interferometry of a Rydberg-Dressed Spin Lattice, Nat. Phys. 12, 1095 (2016).

[73] V. Lienhard, S. de Léséleuc, D. Barredo, T. Lahaye, A. Browaeys, M. Schuler, L.-P. Henry, and A. M. Läuchli, following article, Observing the Space- and TimeDependent Growth of Correlations in Dynamically Tuned Synthetic Ising Models with Antiferromagnetic Interactions, Phys. Rev. X 8, 021070 (2018). 